\title{
Sub-critical transitions in coupled map lattices
}

\author{
P. Manneville and L.A. Zepeda Núnez
}

Hydrodynamics Laboratory

École Polytechnique

91128 Palaiseau, France

(e-mail: paul.manneville@polytechnique.edu)

\begin{abstract}
Sub-critical transitions between two collective regimes with different periods have been observed in a 4-dimensional hypercubic lattice of coupled logistic maps. Intermittent switching between the two regimes takes place in the upper part of the bifurcation diagram while systematic decay with exponentially distributed transient lifetimes is observed in the lowest part of the coexistence region. Statistical findings can be interpreted in terms of macroscopic quantities (measuring global properties) escaping from potential wells in the presence of noise induced by chaos in the microscopic dynamics (local variables).
\end{abstract}

Keywords: Coupled Map Lattices, Sub-critical transition, Escape from attractors.

\section{Introduction}

Large assemblies of interacting nonlinear units give examples of complex systems in which collective behaviour emerges, characterised by the ordered evolution of averaged quantities in spite of strong chaos persisting at the scale of the individual units. The acronym NTCB, meaning non-trivial collective behaviour, has been coined to label this dynamics, emphasising the fact that conventional thermodynamic arguments seem to rule them out. In the present study, we consider coupled map systems [4] in the form:

$$
X_{j}^{t+1}=\frac{1}{2 d+1} \sum_{j^{\prime} \in \mathcal{V}_{j}} f_{r}\left(X_{j^{\prime}}^{t}\right),
$$

where $t$ denotes (discrete) time and $\mathcal{V}_{j}$ the neighbourhoods of sites $j$ at nodes of a $d$-dimensional hypercubic simple lattice. Here, $\mathcal{V}_{j}$ is limited to nearest neighbours and the local evolution is governed by the logistic map $f_{r}(X)=r X(1-X)$. Interesting values of $r$ lie beyond the accumulation point of the sub-harmonic cascade $(r>3.57)$. Such models have already been studied in various space dimensions [1]. They are known to display NTCB for $r$ somewhat below the crisis point $r_{\max }=4$. The size of the system as measured by the number of sites $N=n^{d}$, where $n$ is number of sites along a dimension, is an important parameter and, remarkably, NTCB is better and better defined as $N$ increases. Accordingly, it is believed that the asymptotic evolution in the infinite-time limit is accounted for by a genuine attractor in the infinite-size limit. 
Bifurcation between different NTCB has been observed upon varying $r$ and the supercritical period-doubling cascade is well documented [2]. We focus here on the subcritical bifurcation between two NTBC regimes T2 and T4, with respective periods 2 and 4 , coexisting in the range $3.88<r \leq 4$, and take this situation as an analogue of that existing in the von Kármán swirling flow between counter-rotating disks which display subcritical transitions between two - yet unexplained from first principles - turbulent regimes [5]. Only preliminary results are presented; a more complete analysis is in progress.

\section{Results}

\subsection{Overview}

NTCB is identified from the evolution of the mean value of the $X_{j}^{t}$ over the lattice at time $t, \mathcal{X}^{t}=N^{-1} \sum_{j} X_{j}^{t}$. When starting from a distribution of site states uniform over $[0,1]$, a periodic evolution with period 4 (transient state T4') is rapidly obtained, within 20 to 40 iterations, which later turns into a more sustained period 2 (state T2) or to a period 4 (state T4), depending on the precise value of $r$ and the size $n$ of the system, see Fig. 1. The differences
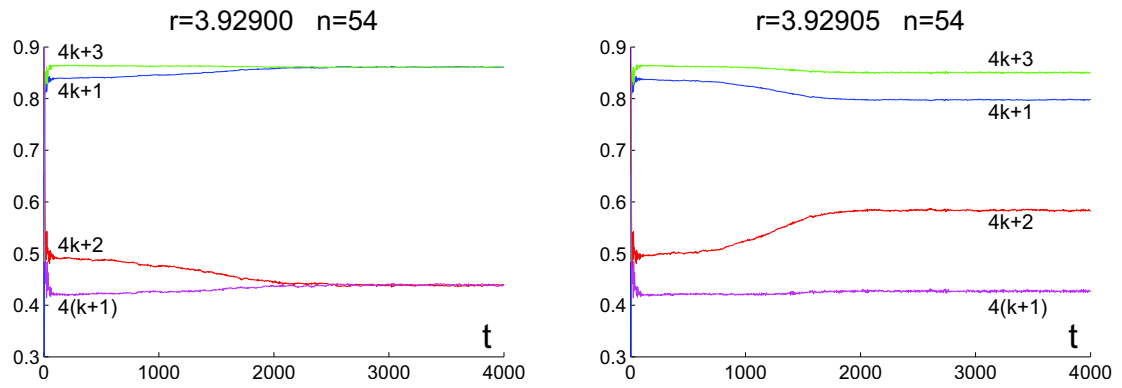

Fig. 1. Time series of $\mathcal{X}^{t}$ for $n=54$ and different values of $r$ with initial conditions uniformly distributed over [0,1]. Color indicates time modulo 4 ; blue: $4 k+1$, red: $4 k+2$, green: $4 k+3$, magenta: $4(k+1)$. Regime T4' here apparent for $t<1500$ evolves into regime $\mathrm{T} 2$ for $r$ below $\approx 3.93$, here for $r=3.929$ (left) and into regime T4 otherwise, here for $r=3.92905$ (right).

between these states are not obvious from snapshots of local configurations and the detailed mechanisms that govern the different macroscopic states are not known. In particular, the mean-field theory obtained by replacing variables by mean values and neglecting correlations is unable to explain the emergence of such collective states. With $n=54$, regime T2 is systematically obtained in the long run for $r<3.9290076$ and regime T4 in the opposite 
case. Transients are usually long but their lengths depend sensitively on the initial configurations.

Bifurcation diagrams obtained for the 4d-CLML are presented in Fig. 2. As usual in a subcritical case, the experimental protocol has to be precisely specified. Here we consider systems with linear size $n=27$ and mostly
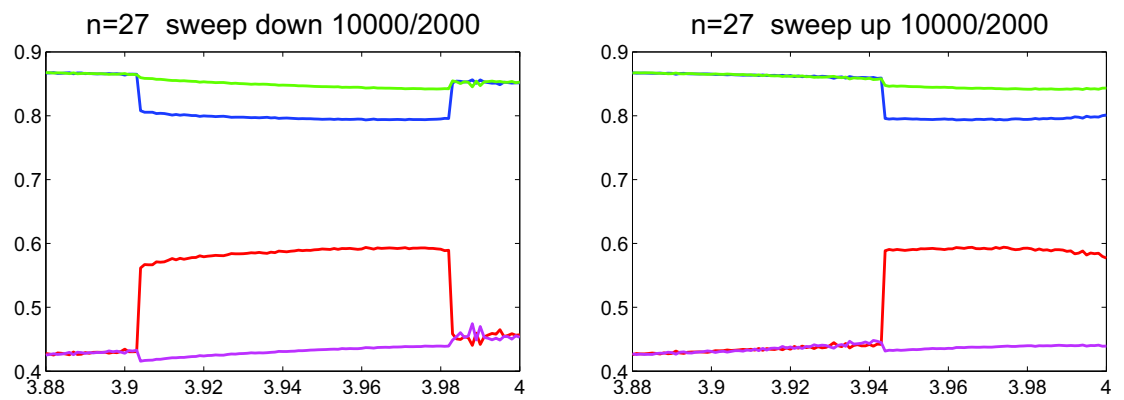

Fig. 2. Bifurcation diagrams obtained for $n=27$ by sweeping $r$ up from 3.88 (left) and down from 4.00 as described in the text. Same color coding as in Fig. 1

use helical boundary conditions. ${ }^{1}$ Other kinds of boundary conditions, e.g. periodic, give the same results provided that the system is large enough. Parameter $r$ is adiabatically swept up (down), starting at $r=3.88$ (4.00), by increasing (decreasing) it steadily by steps $\delta r=10^{-3}$. Adiabatic here means that $10^{4}$ iterations are performed at given $r$ (the value reported is an average over the last $210^{3}$ iterations) and that the state at the end of the series of is used as initial condition for the next value of $r$. Hysteresis is obvious since, according to this protocol, in the lower part of the diagram, the transition $\mathrm{T} 2 \rightarrow \mathrm{T} 4$ is observed at $r \approx 3.94$ when sweeping $r$ up and the reverse transition $\mathrm{T} 4 \rightarrow \mathrm{T} 2$ at $r \approx 3.90$ when sweeping it down. A transition $\mathrm{T} 4 \rightarrow \mathrm{T} 2$ is observed at $r \approx 3.98$ in the upper part of the diagram upon decreasing $r$ while the T2 state is maintained up to $r=4$ when increasing $r$. For $n=54$, using the same protocol, the up-sweep $\mathrm{T} 2 \rightarrow \mathrm{T} 4$ bifurcation is not observed and the $\mathrm{T} 2$ regime obtained for $r=3.88$ is kept up to $r=4$. The low- $r$ part of the down-sweep bifurcation diagram is the same as for $n=27$ when starting from an initial condition belonging to the $\mathrm{T} 4$ regime, i.e. the $\mathrm{T} 4 \rightarrow \mathrm{T} 2$ transition takes place at $r \approx 3.90$, but no $\mathrm{T} 2 \rightarrow \mathrm{T} 4$ transition is observed when starting from an initial condition belonging to the T2 regime which is kept down to $r=2.88$. In [1], back with $n=27$ but with a slightly different protocol $\left(\delta r=2.10^{-3}\right.$, 2000 iterations with the last 500 displayed), a different coexistence range was

\footnotetext{
$\overline{1}$ The lattice is arranged as a one-dimensional array of size $n^{4}$ with periodic boundary conditions. The coupling then reads $\left(Y_{j}+Y_{j+1}+Y_{j-1}+Y_{j+n}+Y_{j-n}+Y_{j+n^{2}}+\right.$ $\left.Y_{j-n^{2}}+Y_{j+n^{3}}+Y_{j-n^{3}}\right) / 9$ for $j=1, \ldots, n^{4}$, where $Y_{j}=f_{r}\left(X_{j}\right)$.
} 
obtained, $r \in[3.90,3.97]$ with a transition $\mathrm{T} 4 \rightarrow \mathrm{T} 2$ at $r \approx 3.97$ when sweeping up.

These results clearly motivate a detailed study of metastability and associated transient behaviour. In the following we first consider metastability at $r=4$ in order to explain the difference between early results and those reported in Fig. 2 in the upper part of the bifurcation diagram $(\$ 2.2)$ and next turn to a study of transients at the $\mathrm{T} 4 \rightarrow \mathrm{T} 2$ transition observed in its lower part (§2.3).

\section{$2.2 \quad$ Intermittent switching at $r=4$}

Size effects are most easily observable at $r=4$ as seen in Fig. 3 which displays time series of $\mathcal{X}^{t}$ for systems with $n=16,19,23,27$, with numbers of sites increasing by factors of 2 from $6.510^{4}$ to $5.310^{5}$. The T4 regime corresponds to 4 bands and the T2 regime to 2 bands only with the $4 k+3$ and $4(k+1)$ series partly masking the $4 k+1$ and $4 k+3$ series. For $n=16$, switchings between $\mathrm{T} 4$ and $\mathrm{T} 2$ are frequent and it may happen that a phase change occurs upon return to the T4 regime, e.g. after the T2 episodes at $t \approx 1.310^{5}, \approx 1.4210^{5}$ and $\approx 1.8210^{5}$. For $n=19$, switchings are less frequent, even less for $n=23$, and for $n=27$ regime T2 seems sustained (notice the change of time scales for $n=23,27)$.
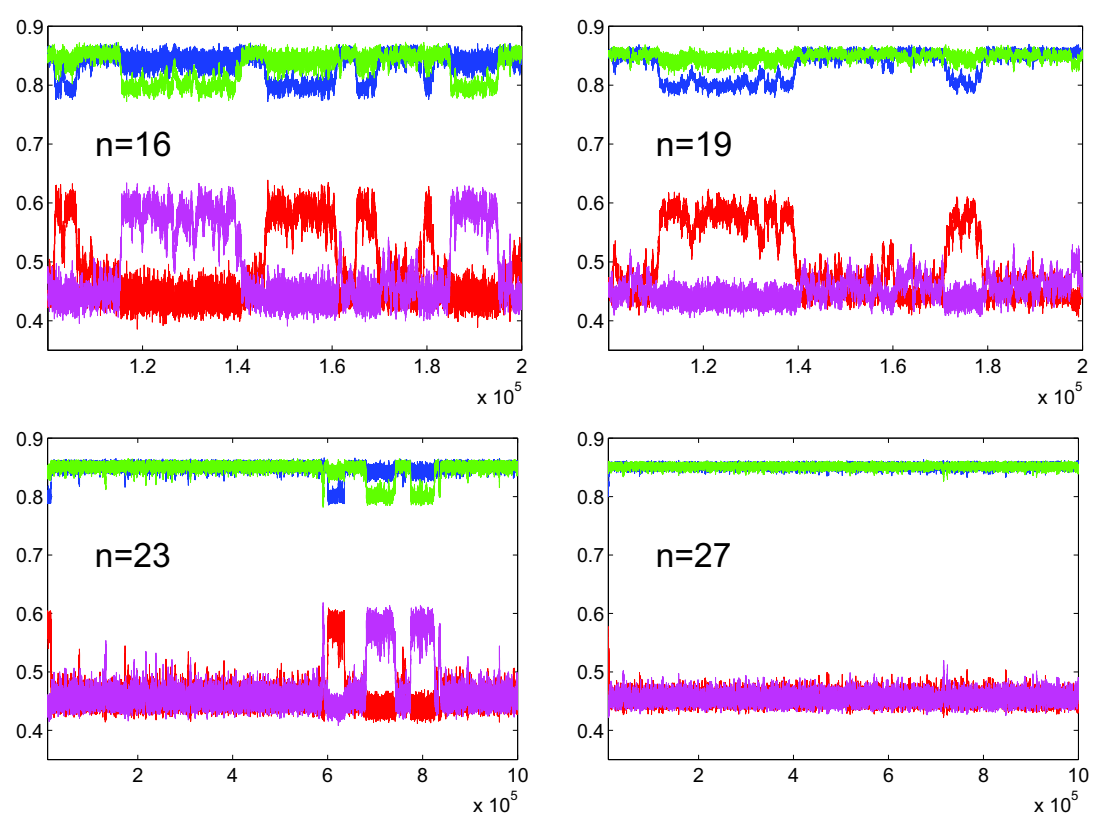

Fig. 3. Time series of $\mathcal{X}^{t}$ at $r=4$ for systems of increasing sizes. Same color coding as in Fig. 1. 
For $n=54$, the T4 regime obtained from uniformly distributed initial conditions does not turn to T2, and the T2 regime obtained at the top end of the up-sweep never decays into T4 during experiments of more than $10^{7}$ iterations. It can be concluded that the switch is triggered by a noise of variable intensity function of the size. The dynamics can thus be recast in terms of standard escape problem [3]. A detailed quantitative study is under way to support the idea to be discussed in $\S 3$ that the T2 and T4 regimes lie at minima of a 2-well potential separated by a barrier and that the lifetimes in each regime is governed by Arrhenius-like processes where the noise intensity plays the role of a temperature.

\subsection{Transients at the $\mathbf{T} 4 \rightarrow \mathbf{T} 2$ transition for quench experiments from $r=3.98$ to $r \simeq 3.90$}

Which is the most stable state in the range $3.90<r<4.0$ is not yet known. It seems however clear that the T4 regime is not observed when $t \rightarrow \infty$ and $n$ sufficiently large as soon as $r$ is substantially smaller than 3.93 (see $\S 2.1$, Fig. 1) and that the $\mathrm{T} 4 \rightarrow \mathrm{T} 2$ decay is a good example of relaxation at a discontinuous (first-order) transition in a high-dimensional dynamical system. The statistics of waiting times in the T4 state before decay into the T2 state have been studied in series of quench experiments where the system is prepared in a T4 state at some initial value $r_{\mathrm{i}}$ of $r$ that is next abruptly changed to some final value $r_{\mathrm{f}}<r_{\mathrm{i}}$ where the time asymptotic regime is expected to be T2. Quantitative results do not depend on the value of $r_{\mathrm{i}}$ provided that it is sufficiently high above the upper boundary, 3.93, of the critical zone. In practice we have taken $r_{\mathrm{i}}=3.98$ and studied the range $3.88<r<3.91$ for different values of $n$. Results for $n=27(N=531441)$ are presented in Fig. 4. From 200 to 400 transients were examined, the length of a transient was determined from the sudden decrease of the quantity $\mathcal{X}^{4 k+2}-\mathcal{X}^{4(k+1)}$ which is finite in regime $\mathrm{T} 4$ and statistically zero in regime
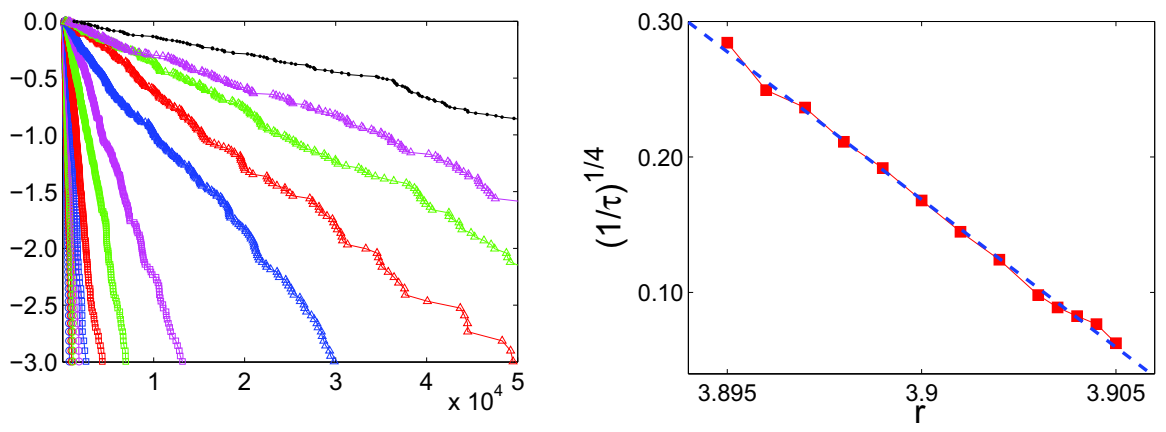

Fig. 4. Left: Distribution of transient life-times in quench experiments for $n=27$ and $r_{\mathrm{i}}=3.98$ and various values of $r_{\mathrm{f}}$ from 3.905 (upper curve with black dots) down to 3.985. Right: Variation of characteristic time with $r_{\mathrm{f}}$. 
T2. The left panel of Fig. 4 displays the logarithm of fraction of systems still in regime T4 at time $t$ as a function of $t$ itself. The distribution of lifetimes of transients displays an exponential tail in the form $\mathcal{P}\left(t^{\prime}>t\right) \propto \exp (-t / \tau)$, where $\tau$ is the corresponding characteristic time. Parameter $\tau$ is a function of $r$ and, as seen in the right panel of the figure, its variation is not incompatible with an algebraic divergence of the form $\tau \sim 1 /\left(r_{\lim }-r\right)^{4}$ with $r_{r m l i m}>3.91$.

\section{Discussion}

Bifurcation theory applied to the evolution of the macrovariable $\mathcal{X}$ accounting for NTBC is an appealing framework provided that it is corrected by the introduction of noise due to the chaotic evolution of microvariables $X_{j}$. Let us assume that the dynamics of the system can be reduced to a one-dimensional iteration for an effective order parameter $\mathcal{Z}$ able to distinguish T2 from T4. A suitable expression would be $\mathcal{Z}^{t+1}=(1+a) \mathcal{Z}^{t}-\left(\mathcal{Z}^{t}\right)^{3}+b$, but noticing that the global dynamics is slow (tumbling from one regime into the other takes hundreds of iterations) and introducing noise, one should rather consider the equivalent Langevin equation

$$
\frac{\mathrm{d}}{\mathrm{d} t} \mathcal{Z}=a \mathcal{Z}-\mathcal{Z}^{3}+b+\zeta(t)
$$

The deterministic part of this equation derives from a two-well potential that would display a single minumum corresponding to regime T2 below $r=3.88$ and a supplementary minimum for T4 above. A consistent interpretation of the experiments would then follow from the observation that the variance $\sigma^{2}$ of the noise varies with the system size as $N^{-1}[1]$ and from appropriate variations with $r$ of parameters $a$ and $b$ in (1). Work is in progress to turn this conjecture into quantitative predictions, which could also shed some light on the statistics of transients observed in discontinuous hydrodynamical turbulent-turbulent transitions [5].

\section{References}

1.H. Chaté and P. Manneville. Collective behaviors in spatially extended systems with local interactions and synchronous updating. Prog. Theor. Phys., 87:1-60, 1992.

2.H. Chaté, A. Lemaître, Ph. Marcq, and P. Manneville. Non-trivial collective behavior in extensively-chaotic dynamical systems: an update. Physica A, 224:447-457, 1996.

3.N.G. Van Kampen. Stochastic Processes in Physics and Chemistry. NorthHolland, Amsterdam, 1983.

4.K. Kaneko. The Theory and Application of Coupled Map Lattices. Wiley, New York, 1993.

5.F. Ravelet, L. Marié, A. Chiffaudel, and F. Daviaud. Multistability and memory effects in a highly turbulent flow: experimental evidence for a global bifurcation. Phys. Rev. Lett., 93:164501.1-4, 2004. 